\title{
A multi internal standard calibration approach for the automated and robust analysis of wine volatiles by headspace Solid Phase Microextraction and further Gas Chromatography-Mass Spectrometry
}

\author{
P. Herrero, J. Zapata, J. Cacho, Vicente Ferreira \\ Laboratorio de Análisis del Aroma y Enología (LAAE) \\ Instituto de Investigación en Ingeniería de Aragón (I3A) \\ Universidad de Zaragoza, Dpto de Química Analítica, Calle Pedro Cerbuna 12, 50009, Zaragoza, Spain. \\ Tel.+34-97676100 Ext.3328, Fax+34-976761292, e-mail: paulahp@unizar.es
}

\begin{abstract}
Head space solid phase microextraction (HS-SPME) is a solvent-free technique that allows an almost complete automatization and getting amazing sensitivities. The hidden risk of SPME lies in the fact that as the amount of analyte extracted is very low; it is extremely sensitive to any experimental parameter that may affect the liquid-gas and gas-solid distribution coefficients. Our aims are to measure the relative weight of these factors on the lack of accuracy, and to design a robust calibration system able to avoid or limit their effects.

For the first goal, synthetic but real-like wines containing a fixed amount of selected analytes (70) and variable amounts of ethanol, non-volatile constituents and major volatile constituents were prepared following a 3-Factor complete Factorial design. The study of the relevance of the Factors carried out by analysis of variance (ANOVA) and by Principal Component Analysis revealed that the levels of major volatile constituents affected the extraction of most analytes, while ethanol and matrix affected particularly low volatile compounds. Lipophilic esters are most influenced by major volatile compounds, while acids, phenols and lactones are affected by the non-volatile matrix.

13 different internal standard compounds belonging to different chemical classes were used in the calibration experiment. This was similar to the aforementioned experiment, but including as well 5 different concentration levels. In 29 out of 65 cases, a single internal standard provided a robust calibration guaranteeing an accuracy better than 10\%, while in others a Partial Least Square Regression analysis was run in order to find a model able to provide maxima accuracy. Satisfactory models in terms of precision, linearity and recovery could be built for 30 other compounds, so that the method can quantify up to 59 relevant wine volatile compounds.
\end{abstract}

